\title{
Public health aspects of visceral leishmaniasis in Montenegro"
}

\author{
Bogdanka Andric ${ }^{\#}$, Dragica Terzic, Brankica Dupanovic, Aleksandar Andric \\ University Medical School of Montenegro, Podgorica, Montenegro \\ Email: "bogdankaandric0@gmail.com
}

Received 28 October 2013; revised 28 November 2013; accepted 5 December 2013

Copyright (C 2013 Bogdanka Andric et al. This is an open access article distributed under the Creative Commons Attribution License, which permits unrestricted use, distribution, and reproduction in any medium, provided the original work is properly cited.

\section{ABSTRACT}

Leishmaniasis belongs to the parasitic communicable zoo noses, caused by members of Leishmania species. The infected phlebotomies sand fly carries the parasites, which cause the different forms of disease. Retrospective/prospective review of records for documented cases of visceral leishmaniasis (VL) in period from 1992 to 2012 in Montenegro shows 83 diagnostic cases, and $1(1.20 \%)$ case with dermal leichmaniasis. with 3 (3.61\%) deaths cases. Analyses of age show: 36 (43.37\%) children and $47(56.63 \%)$ adults. Examinations are based on epidemiological, clinical, hematological, patohystological and serological investigations. Infection can be sub-clinically or clinically manifested with acute, sub-acute, and chronic type. Incubation in clinically manifested infections ranges from several weeks to several months. In our study, the prevalence of general infective syndrome is registered in all of 83 manifested cases $(100 \%)$. Enlarged spleen in 79 (95.18\%) cases, enlarged liver in $37(44.57 \%)$ cases, anemia in $49(59.04 \%)$ cases, pancytopenia in 32 (38.55\%) cases, and increased activity of serum aminotransferases in $37(44.57 \%)$ cases. The diagnosis was confirmed by an analysis of bone marrow biopsy material by direct microscopy of serial sections colored by Romanowski and Giemsa s staining, and by immune-biochemical methods. Serological diagnostic is confirmed by using agglutination test. In Montenegro (in humans and dogs) two types of leishmania (L) ( $L$. donovani, $L$. infantum were presented). As to therapy treatment, the common treating is with antimony drugs: glucantime is relatively satisfactory for a long time. During 2008 there were registered

\footnotetext{
Non-existence of the conflict of interests.

" Corresponding author.

Specialist for infectious diseases, expert to zoonotic infectious diseases. Professor for infectious diseases in University Medical School of Montenegro.
}

cases not responding to the therapy and those were manifested with relapses after therapy. In the first line of therapy, we used meglumine antimony (Glucantime) in $78(93.97 \%)$ patients. Resistence developed in $7 \mathbf{( 8 . 9 7 \% )}$ during treatment, and relapse occurred in $5(6.41 \%)$ patients. It was $1(1.20 \%)$ patient treated with Miltefosine the one who had a relapse, and with Amphotericin B (Ambisome) 4 (4.82\%) patients.

Keywords: Leishmaniasis; Resistance; Severity; Diagnostic Difficulties

\section{INTRODUCTION}

Leishmaniasis, parasitic infectious disease, caused by trypanosomatides from the genus Leishmania spp. widely expanded in the world. Transmission occurs in 88 tropical and subtropical countries where the sand fly vectors are present. According to the World Health Organization (WHO) data, approximately 350 million people worldwide, are at risk of infection [1-3]. In endemic areas the numerous natural reservoirs of parasites are present. Dog is the main reservoir, responsible for human infections $[2,3]$. The incidence and the prevalence of the disease cannot be precisely defined. Visceral leishmaniasis (VL) cases increase around the World, with approximately 500,000 cases per year [2,4,5], being a significant and dangerous opportunistic infection in people with acquired immune deficiency $[6,7]$.

Montenegro is an endemic area for VL (Kala azar). Natural condition and geographical position (Mediterranean area) helps to the existence of the disease $[8,9]$. Misbalance of Eco-system, increase of vector density and reservoirs of Leishmania spp., are causes of the expansion of the primary endemic focus, and number of infected cases. This also includes co-infections among different species of leishmania, as well as leishmania 
with other agents of vector borne diseases (VBD) complex in common endemic areas [10,11].

The life cycle of the parasite is relatively simple, whereas the immune-pathogenic mechanisms of infection are very complex and variable [1].

Leishmania parasite lives a dual form life cycle, a flagella promastigote or amastigote forms. The promastigote are found in the vectors and are injected into the mammalian host during vector s blood meal $[12,13]$. In the mammalian host, leishmania agents are obligate intracellular parasites of macrophages, dendrite cells and/ or neutrophils where are differentiate into amastigotes and can have different host cells and organ tropism, infecting superficial cells (dermal leishmaniasis—skin sores), mucocutaneous leichmaniasis [14], or visceral cells (visceral leichmaniasis) $[15,16]$.

Clinical manifestations of leishmaniasis depend of the outcome of the complex interactive relation of the parasites and immunological defense of the host. Every infection doesn't lead to the clinically manifested disease, however the persistence of live parasite long after the infection itself, put it into the group of important opportunistic agents $[17,18]$.

Infection can be sub-clinically or clinically manifested with acute, sub-acute and chronic phase. Incubation in clinically manifested infections ranges from several weeks to several months. The general weakness and exhaustion are followed by organ manifestations, and with changes in the blood [19,20].

Etiological diagnosis of the disease is not easy. It is possible to detect it by examination or by culture samples obtained either by biopsy of the bone marrow, spleen, liver or lymph glands. Serological diagnosis is made on identification of specific antibodies that can be important for diagnostics itself, but which are not protective [2123].

Over decades, five-valents salt of antimony (Sbv) has been used for treatment of visceral leishmaniasis: sodium stibogluconate (Pentostamin) and meglumine antimonate (Glucantime). In recent years in many endemic areas a resistance to antimony drugs has been observed. Alternative medications that can be used are amphotericin $B$ deoxycholate and pentamidin isethionate, but they are more toxic [24-27].

Treatment of leishmaniasis in human immunodeficiency viral infection (HIV patients) starts with an initial parasitological cure with five valents salt of antimony, amphotericine B. deoxycholate and lipid-associated amphotericin $B$. Secondary prophylaxis is conducted with liposomal amphotericin-B, miltefosine [28].

Repellents, protective nears and protective clothes, vector control and reservoir elimination (the dogs) are used for prevention. The vaccine is still in the research phase.

Aim: Study presents epidemiological, clinical, labora- tory characteristics of leishmaniasis in Montenegro for the period from 1992-2012, important for diagnosis, prognosis and public health aspects of disease.

\section{METHODOLOGY}

This is retrospective-prospective study in Montenegro, for the period from period of 1992 to 2012. The study included epidemiological, clinical and laboratory diagnostics, and specific microbiological and pathohistology analysis. Epidemiological dates are processed in cooperation with the epidemiology department and referred to the place of residence, owning a dog.

Clinical data were related to the presence of fever, liver and spleen enlargement. Laboratory diagnosis included the values of white and red cells, blood platelets, biochemical parameters of the liver function, lactate dehydrogenasis (LDH). Specific microbiological analysis were etiologically confirmed through test of agglutination serologically, and analysis of bone marrow biopsy by direct microscopy of serial sections colored with the Romanowski and Giemsa - s staining, and immune-biochemical methods (Tat, CD34, CD117, CD15, Glycophorin A, CD31, CD79a, CD20, CD3, CD45RO, CD38, kappa, lambda, IgG, IgM, IgA, CD68). In Montenegro (in humans and dogs) two types of leishmania-L. donovani and $L$. infantum are presented.

Analysis of serums from different parts of Montenegro is done using the Indirect of 1500 dogs' Immune fluorescence (IIF) method. The results indicated the high infectiveness of dogs with leishmania parasites in the examined sample in endemic areas.

\section{RESULTS}

This are the results found in retrospective/prospective investigation of leishmaniasis in period from 1992 to 2012 in Montenegro. Visceral leishmaniasis (kala-azar) was diagnosed in 83 cases, and $1(1.20 \%)$ case had dermal leichmaniasis, with 3 (3.61\%) deaths. Analyses of ages shows different age cohort: 36 (43.37\%) children, and 47 (56.63\%) adults. Distribution of age among children population presented: 7 (19.45\%) cases aged of 0 4 years, 29 (80.55\%) cases aged from 5 - 15.

According to the geographic area where the disease was diagnosed, the expansion of kala-azar endemic focus in Montenegro is evident. Initially it included the narrow area of the southern part of the Montenegrin coast (between Bar and Ulcinj). According to the collected data, these areas now include the entire costal area of Montenegro from Ulcinj to Herceg Novi, the Scadar lake area, including Podgorica, Cetinje, and even some northern parts of Montenegro. Incidence rates of pediatric cases in 2001 year are presented in the Table 1.

The obtained results indicated the high infectiveness 
Table 1. Incidence rates of pediatric cases in Montenegro and countries in encircle 2001.

\begin{tabular}{ccccc}
\hline Countries & Montenegro & Albania & Italy & Macedonia \\
\hline Incidence & $0.9 / 100,000$ & $7 / 100,000$ & $0.3 / 100,000$ & $0.4 / 100,000$ \\
$\begin{array}{c}\text { Percentage } \\
\text { frequency }\end{array}$ & 67 & 86 & 50 & 63 \\
\hline
\end{tabular}

of dogs with leishmanias, which is $83 \%$ in examined sample serum of 1500 dogs.

The significance and interest in phlebotomies species is growing along with the increase in frequency of numerous viral, bacterial and parasitic infections in the group of vector borne diseases (VBD), in which phlebotomies are reservoirs and vectors of infections. Evolution adaptability, continuously allow phlebotomies to significantly expand their potential of vector and direct participation in expansion and causing VBD, which is in correlation with global changes of ecologic environment and natural base of VBD. This is already evident in practice and there is a tendency of further growth. Epidemiological studies conducted in period 1996-1999 and 2003 in the endemic area of kala-azar (the southern part of the Montenegrin coast) on 4770 samples of phlebotomies [8] showed presence of 5 kinds: Ph. Perfiliews (1\%), Sergentomya minuta (12\%), Ph. papatasi (11\%), Ph. neglecticus (60\%). Predominantly Ph. neglecticus is mostly found in indoor areas. It is assumed that the main vector in the Bar-Ulcinj region and in the northern-east Mediterranean is L. infantum. As in the prior studies, the number of phlebotomy in resistential facilities was increased. Examination [8], show that for the first time in Montenegro the kind $\mathrm{Pl}$. kandelaki, which is pertinent to Middle Asia and the neighboring Albania was identified in Montenegro as vector of $L$. infantum.

In our study, clinical diagnosis in manifest cases was made on the basis of non specific, multi-systemic manifestations.

Prevalence of general infective syndrome (fever, exhaustion, musculoskeletal pain, headache) was registered in all 83 cases (100\%), enlarged spleen in 79 cases (95.18\%), and liver in 37 (44.57\%) cases. Laboratory analysis showed presence of anemia in 49 (59.04\%) cases, pancytopenya in $32(44.57 \%)$ cases, increased activity of serum aminotransferases in 37 (44.58\%) cases

\section{(Table 2).}

Patohystological diagnosis is based on bone marrow biopsy by direct microscopy of serial sections colored by the Romanowski and Giemsa s stained in 10 cases (Figure 1), and by immune-biochemical methods (TdT, CD34, CD117, CD15, Glycophorin A, CD31, CD79a, CD20, CD3, CD45RO, CD38, kappa, lambda, IgG, IgM, IgA, CD68).

As to therapy treatment, the common treating with pentavalent antimony: glucantime was relatively satis-
Table 2. Clinical presentations of kala-azar in our study N-83.

\begin{tabular}{ccc}
\hline Symptoms and signs & $\begin{array}{c}\text { Number } \\
\text { of cases }\end{array}$ & $\begin{array}{c}\text { Percentage } \\
\text { frequency }\end{array}$ \\
\hline $\begin{array}{c}\text { General infective syndromes: } \\
\text { fever, excaution, muscle-sceletal pain etc. }\end{array}$ & 83 & 100 \\
Enlarged of spleen & 79 & 95.18 \\
Enlarged of liver & 37 & 44.57 \\
Anaemia & 49 & 59.04 \\
Pancytopenia & 32 & 44.47 \\
Increased serum aminotransferases & 37 & 44.58 \\
\hline
\end{tabular}
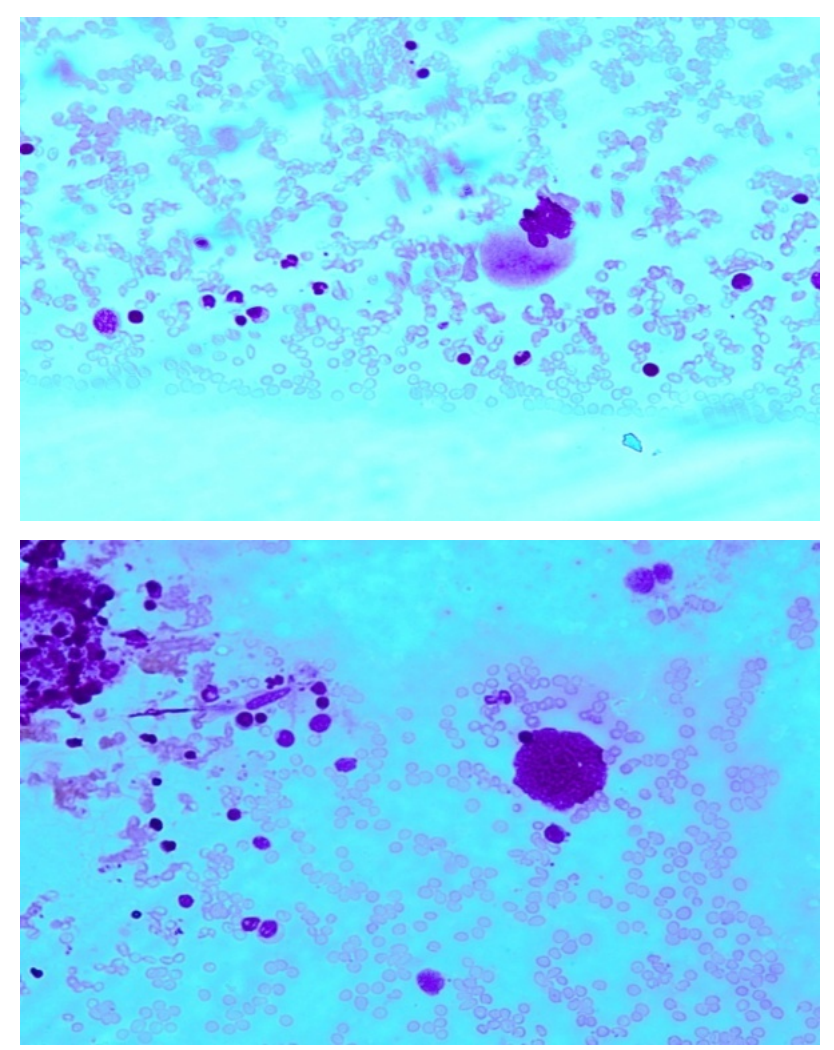

Figure 1. Bone marrow biopsy preparates colored by Romanovski, present amastigote forms of leichmania parasites extracellular and in leucocytic cells. Original preparates thanks to Prof. Mileta Golubovic.

factory over long period of years. During 2008 the first 7 $(8.97 \%)$ cases that did not respond to therapy treatment with glucantime were registered, so the treatment with liposomal amphotericin B was continued. The other 5 (6.41\%) cases resistable to glucantime were registered in 2009. In both cases the therapy initiated with a glucantime, and was repeated after one month. Due to the progressive course of the disease in one case therapy with miltefosine doesn't prove good results. In 4 cases the treatment with liposomal amphotericin B was continued. The patient is still observed considering the persistent spleen enlargement. 


\section{DISCUSION}

In the period from 1992-2012 in Montenegro 83 cases of manifested kala-azar were registered. In the sample children population is presented with 36 cases. Based on the number of registered cases a conclusion may be drawn that the problem of this parasitic disease does not have a high significance. Viewed from the total number of inhabitants of Montenegro, the number of diseased is statistically significant, with 3 patients on 646,000 inhabitants per year. Statistical trend making predictions classifies it into infections which in the near future, when drastic changes of eco-system are expected due to the effects of globalization, may come to the focus of infective and veterinary pathology $[10,11]$.

Since 1998 clinical studies, Projects-Transmissible Vector Borne Zoonoses in Montenegro of the University Medical School of Montenegro [10], the International Project: SEE-ERA.NET Pilot Joint Call, and some others, have pointed out to the potential significance of this severe parasitic disease and the necessary measures were proposed. Visceral leishmaniasis is a severe parasitic disease from the group of VBD, both due to the pathogenic potentials of etiologic agents and to the fact that in the infected organism it leads to an immunodeficiency with an uncertain outcome [28].

Complex immunogenetic mechanisms of the infection, place the macrophages into the focus of leishmaniasis infection $[29,30]$.

In vitro studies using murine and human mononuclear phagocytes indicate that promastigote can be related to numerous macrophage receptors. Two surface promastigote molecules have been recognized as directly responsible for parasite $63 \mathrm{kDa}$ neutral proteases adherence-gp63 [29,30]. As well as lipophospoglycan (LPG) [31,32]. As to the host, the adherence was mediated with numerous and various macrophage receptors including complement CR-1 and CR-3 receptors, manosa-fructosa receptors and many others.

It has been proved that both human and murine macrophages can activate interferon-y (INF-y) or cytokines to kill the intracellular amastigotes in such way that INF-y subsequently leads to activation of numerous oxidative and non-oxidative microbicidal mechanisms. Macrophage anti-leishmanial microbicidal mechanisms include the action of Tumor Necrosis Factor-alpha (TNF-alpha) and due to the INF-y they influence the induction of nitric-oxide synthase. In addition, on the surface of the cells specific anti leishmanial substances can be activated in the direct contact with CD4 + T lymphocytes with the assistance of TNF-alpha [33,34].

The numerous leishmanial immunology and immunogenetic studies on rats as models, showed that in $L$. donovani numerous substances that control resistance of intracellular pathogens have been discovered. These genes are also found in salmonella typhimurium and micobacterium spp [35]. Genetic determinants of human leishmaniasis are not sufficiently defined.

Manifestations of the disease and the course of the infection vary. Numerous studies in Africa [36] and Brazil $[37,38]$ showed that in small number of people infected with $L$. donovani or $L$. infantum/chagasi, progressive visceral leishmaniasis occurs. In smaller number the course of the infection is asymptomatic or with minor symptoms as a self-limiting infection.

It was obvious that infections, re-infections and protections, where associated with leishmania specific CD4 + T lymptocytes and Th- 1 subtype of immune response, as well as with production of INF-y on leishmania antigens $[39,40]$. Leishmania promastigote is capable of inhibiting production of Il-1 and TNF-alpha as a response to parasite presence [40]. In humans, it is typical that immunity to the $L$. donovani activation leads to hypersensitive skin reaction.

Visceral leishmaniasis is a multispectral syndrome. The largest number of infections is asymptomatic, which was shown both in screenings and in studies of a defined endemic area in the south part of the Montenegrin coast. Therefore the disease is considered as self limited and benign.

In Montenegro the single database does not exist and there's a lack of insight as to the real significance of this disease for the national pathology. Epidemiologically indicators of expanding of endemic focus are evident in clinical examinations of infection control and veterinary services in Montenegro (based on registration of number cases that got infected out of the registered endemic area as well as based on screening of dogs infected with this parasite as well as on reports on occurrence of clinical manifestations suspected on kala-azar in cattle in the northern parts of Montenegro) [11,12].

If we accept the known fact on persistence of live leishmanias long time after the infection, based on the recent facts on immunopathogenic potentials of parasites, it is obvious that the spectrum of clinical manifestations of the disease is not always the indicator of the seriousness of the disease. Gradual and progressive disabling of the coded cell immunity to combat the parasite is the essence of the infection, which is also significant for the prognosis of the disease. In our studies we considered theoretical possibilities of mutual action and reciprocal immunologic impact of $L$. donovani and different intracellular agents on the course and the seriousness of the disease from the aspect of their activity as opportunistic agents during clinical studies [36,41]. We haven't registered this co-infections in examined sample of HIV/ AIDS patients (15 cases). The increases of resistance to antimony preparates that have been successfully used 
over years for leishmaniasis treatment prove that they became inefficient. In our series, it led to an alternative treating in 5 patients, with discutable therapeutical effects.

\section{CONCLUSIONS}

Leishmaniasis is a severe parasitic disease in the group of VBD around the world. In our country there is no single database and there's the lack of insight as to the real significance of VL in national pathology. Based on the number of registered cases a conclusion may be drawn that the problem of this parasitic disease does not have a high significance. Viewed from the total number of inhabitants of Montenegro, the number of diseased is statistically significant, with 3 patients per year on 646,000 inhabitants.

Investigations for the period 1992-2012, presents increasing number of documented cases of VL, increased number of clinically manifested syndromes of the disease and increase of the cases resistant to therapy.

Epidemiological studies of the period 1992-1999 presented indicators of expanding endemic area in Montenegro. The veterinary studies showed the high infectiveness of dogs with leishmanias, in $83 \%$ of examined sample serum of 1500 dogs. Investigations of vectors, analyzing 4770 samples of phlebotomies, showed presence of 5 kinds. In the 1993 for the first time in Montenegro the kind's $P l$. kandelaki was identified, as vector of $L$. infantum.

It is very difficult to make the diagnosis of the leishmaniasis. Major part of infected cases was passed in sub clinical manner. New research confirmed that difficulties and prognosis of the outcome of the disease are not obligatory in correlation with clinical manifestations of the disease. The knowledge about persistence of live leishmanias long time after infection is based on the recent proofs on immune pathogenic potentials of parasites. We haven't registered leishmanias as an opportunistic agent in HIV/AIDS co infections, after analyzing a small group of target patients. The assumption is that in the future this type of co-infection might become reality. In additions to this, possibility of coexisting infections between leishmanias and large specter of various complexes of VBD agent's in common endemic areas represents a big problem.

The increase of the resistances to antimony drugs successfully used over years for the treatment of leishmaniasis proved that those became ineffective. In our series, it led to an alternative treating in 5 cases, with ineffective therapeutical results.

Apart from multidisciplinary work, leishmaniasis research priorities should include diagnostics, therapy, vectors' control, and the possibility of the use of vac- cines, particularly in the endemic areas.

\section{REFERENCES}

[1] Freitas-Junior, L.H., Chatelain, E., Kim, H.A. and Siquera-Neto, J.L. (2012) Visceral leishmaniasis treatment: What do we have, what do we need and how to deliver it? International Journal for Parasitology: Drugs and Drug Resistance, 2, 11-19.

[2] Desjux, P. (1992) Human leishmaniasis: Epidemiology and Public Health aspects. World Health Statistics Quarterly, 45, 267-275.

[3] Jeronimo, S.M.B., De Queiroz Sousa, A. and Pearson, R.D. (2000) Leishmaniasis. In: Guerrant, R.L., Ed., Tropical Infectious Diseases, 1, 1095-1107.

[4] Gugushvili, G., Sekhniashvill, E., Lomtadze, Z., Zerekidze, L. and Molashvili, L. (2001) About changes in population of transmissible disease vectors. The collection of works of Research Institute of Medical Parasitology and Tropical Medicine, honored to 75-th Year of its Foundation (December 1999), XXXIII, 29-34.

[5] Rassi, Y., Firousi, R. and Javadian, E. (2000) Position of visceral leishmaniasis vectors in the Kalebar focus, East Azarbaijan Province. Moderres, 3, 9-14.

[6] Alvar, J., et al. (2008) The relationship between leishmaniasis and AIDS-The second 10 years. Clinical Microbiology Reviews, 21, 334-359. http://dx.doi.org/10.1128/CMR.00061-07

[7] Rosenthal, E., Marty, P. and Poiyot-Martin, I. (1995) Visceral leishmaniasis and HIV-1 co-infection in southern France. Transactions of the Royal Society of Tropical Medicine and Hygiene, 89, 159-162.

http://dx.doi.org/10.1016/0035-9203(95)90476-X

[8] Ivovic, V., Depaquit, J., Leger, N., Urrano, A. and Papadopulos, B. (2004) Sandflies (Diphtera, Psyhodidae) in the Bar area of Montenegro (Yugoslavia). 2. Presence of promastigotes in Phlebotomus neglecticus and first record of P. kandelakii. Annals of Tropical Medicine and Parasitology, 98, 425-427.

http://dx.doi.org/10.1179/000349804225003352

[9] Ivovic, V., Chaniotis, V., Vujanic, M., Bobic, B., Nikolic, A., Klun, I., Zivkovic, T. and Djurkovic-Djakovic, O. (2010) Life tables and reproductive parameters of phlebotomus neglecticus tonnor, 1921 (Diphtera, Psychodidae) under laboratory condidions. Archives of Biological Science.

[10] Andric, B. (2002) Clinical features and diagnostics in associated transmisive (Ixodiae) zoonoses. Doctoral dissertation, Medical Faculty University in Novi Sad.

[11] Andric, B., Pajovic, B., et al. (2010) Travel and tropical medicine. Medical School, University of Montenegro, Podgorica, Montenegro.

[12] Oshaghi, M.A., Ravastan, N.M., Javadian, E.A., Mohebali, M., Hajjaran, H., et al. (2009) Vector incrimination of sand flies in the most important visceral leishmaniasis focus in Iran. American Journal of Tropical Medicine and Hygiene, 81, 572-577.

http://dx.doi.org/10.4269/ajtmh.2009.08-0469 
[13] Division of Communicable Disease Prevention and Control, Commuunicable Disease Program, HPC/HCT, PAHO (1994) Leichmaniasis in the Americas. Epidemiological Bulletin, 15, 8-11.

[14] Jeronimo, S.M.B., De Queiros Sousa, A. and Pearson, R.D. (2000) Leichmaniasis. Tropical Infectious Diseases, 94, 1095-1107.

[15] Maltezou, H.C., Siafas, C. and Mavricou, M. (2000) Visceral leishmaniasis during childrod in Southem Grece. Clinical Infectious Diseases, 31, 1139-1143. http://dx.doi.org/10.1086/317455

[16] Ready, P.D. and Redy, P.D. (2010) Leishmaniasis emergence in Europe. Eurosurveillance, 15, 19505.

[17] Evans, T.G. and Parson, R.D. (1988) Clinical and immunological responses following accidental inoculation of Leishmania donovani. Transactions of the Royal Society of Tropical Medicine and Hygiene, 82, 854-856. http://dx.doi.org/10.1016/0035-9203(88)90016-8

[18] Mylero, V., Searle, S. and Blackwell, J.M. (2002) Solute carrier $\mathrm{ll}$ a $1 /$ Slcl $\mathrm{l}$ a $\mathrm{l}$ formely Nrramp l/regulates metabolism and release of iron acquired by phagocytic, but not transferin-receptor/mediated, iron uptake. Biochemical Journal, 363, 89-94. http://dx.doi.org/10.1042/0264-6021:3630089

[19] Murrey, H.W., Berman, J., Davies, R.C. and Saravia, N.G. (2005) Advances in leishmaniasis. Lancet, 366, 15611577. http://dx.doi.org/10.1016/S0140-6736(05)67629-5

[20] Guerin, P.J., Olliaro, P., Sundar, S., et al. (2002) Visceral leishmaniasis current status of control, diagnosis, and treatment, and proposed research and development agenda. Lancet Infectious Diseases, 2, 494-501. http://dx.doi.org/10.1016/S1473-3099(02)00347-X

[21] Garcia, L., Kindt, A., Bermudez, H., Lianos-Cuentas, A. and De Doncker, S. (2004) Culture independent species typing of neotropical Leishmania for clinical validation of a PCR based assay targeting heat shock protein 70 genes. Journal of Clinical Microbiology, 42, 2294-2297. http://dx.doi.org/10.1128/JCM.42.5.2294-2297.2004

[22] Tallada, N., Raventos, A. and Martinez, S. (1993) Leishmania lymphadenitis diagnosed by fine-needle aspiration biopsy. Diagnostic Cytopathology, 9, 673-676. http://dx.doi.org/10.1002/dc.2840090614

[23] Bittenecourt, A.L. and Barral, A. (1991) Evaluation of the histopathological classifications of American cutaneous and mucocutaneous leishmaniasis. Mem. Instituto Oswaldo Cruz, 86, 51-56. http://dx.doi.org/10.1590/S0074-02761991000100009

[24] Alvar, J., Croft, S. and Olliaro, P. (2006) Chemotherapy in the treatment and control of leichmaniasis. Advances in Parasitology, 61, 224-261. http://dx.doi.org/10.1016/S0065-308X(05)61006-8

[25] Nishi, G.S. (2011) Visceral leishmaniasis, experimental models for drug discovery. Indian Journal of Medical Research, 133, 27-36.

[26] Frezard, F., Demicheli, C. and Ribeiro, R.R. (2009) Pentavalent antimonials-New perspectives for old drugs. Molecules, 14, 2317-2336. http://dx.doi.org/10.3390/molecules14072317
[27] Herwaldt, B.L. and Berman, J.D. (1992) Recommendations for treating leishmaniasis with sodium stibogluconate (Pentostam) and review of pertinent clinical studies. American Journal of Tropical Medicine and Hygiene, 46, 296-306.

[28] Peters, B.S., Fish, D., Golden, R., Evans, D.A., Bryceson, A.D. and Pinching, A.J. (1990) Visceral leishmaniasis in HIV infection and AIDS: Clinical features and response to therapy. Quarterly Journal of Medicine, 77, 11011111. http://dx.doi.org/10.1093/qimed/77.2.1101

[29] Russel, D.G. and Wilheim, H. (1986) The involvement of the major surface glycoprotein (gp63) of leishmania promastigotes in attachment to macrophages. Journal of Immunology, 136, 2613-2620.

[30] Etges, R., Bouvier, J. and Bordier, C. (1986) The major surface protein of leishmania promastigotes is a protease. Journal of Biological Chemistry, 261, 9098-9101.

[31] Mc Connville, M.J., Bacic, A. and Mitchell, G.F. (1987) Lipophosphoglycan of leishmania major that vaccinates against cutaneous leishmaniasis contains an alkylglycerophosphoinositid lipid anchor. Proceedings of the $\mathrm{Na}$ tional Academy of Sciences of the United States of America, 84, 8941-8945. http://dx.doi.org/10.1073/pnas.84.24.8941

[32] King, D.L., Chang, Y.-D. and Turco, S.J. (1987) Cell surface lipophosphoglycan of leishmania donovani. Molecular and Biochemical Parasitology, 24, 47-53. http://dx.doi.org/10.1016/0166-6851(87)90114-9

[33] Sypek, J.P. and Wyler, D.J. (1991) Antileishmanial defense in macrophages triggered by Tumor Necrosis Factor expended on CD4+T lymphocyte plasma membrane. Journal of Experimental Medicine, 174, 755-759. http://dx.doi.org/10.1084/jem.174.4.755

[34] Birkland, T.P., Sypek, J.P. and Wyler, D.J. (1992) Soluble TNF and membrane TNF expressed on CD4+T lymphocytes differ in their ability to activate macrophage antileishmania defence. Journal of Leukocyte Biology, 51, 296-299.

[35] Mulero, V., Searle, S. and Blackwell, J.M. (2002) Solute carier Il a L/Slcl I a I formely Nrramp l/regulates metabolism and release of iron uptake. Biochemical Journal, 363, 89-84. http://dx.doi.org/10.1042/0264-6021:3630089

[36] Manson-Bahr, P.E.C. (1989) East African kala azar with special reference to the pathology, prophilaxis and treatment. Transactions of the Royal Society of Tropical Medicine and Hygiene, 53, 123-127. http://dx.doi.org/10.1016/0035-9203(59)90060-4

[37] Badaro, R., Jones, T.C. and Lorenco, R. (1986) A prospective study of visceral leishmaniasis in an endemic area of Brasil. Journal of Infectious Diseases, 154, 639649. http://dx.doi.org/10.1093/infdis/154.4.639

[38] Evens, T.G., Teixeria, M.J. and Mc Auliffe, I.T. (1992) Epidemiology of visceral leishmaniasis in north-east Brasil. Journal of Infectious Diseases, 166, 124-132.

[39] Russo, D.M., Barral-Netto, M., Barral, A. and Reed, S.G. (1993) Human T-cell responses in leishmania infections. Progress in Clinical Parasitology, 3, 119-144. http://dx.doi.org/10.1007/978-1-4612-2732-8_5

[40] Reiner, N.E., Ng, W., Wilson, C.B., et al. (1990) Modu- 
lation of in vitro monocyte cytokine responses to leishmania donovani. Interferon-gama prevent parasite induced inhibition of Il-1 production and primes monocytes to respond to leishmania by producting both TNF-alpha and Il-1 production and primes monocytes to respond to leushmania by producting both TNF-alpha and Il-1. Journal of Clinical Investigation, 85, 1914-1924.
http://dx.doi.org/10.1172/JCI114654

[41] Alvar, J., et al. (2008) The relationship between leishmaniasis and AIDS-The second 10 years. Clinical Microbiology Reviews, 21, 334-359.

http://dx.doi.org/10.1128/CMR.00061-07 